\title{
Serum Thyroglobulin Levels in Preterm Infants with and without Respiratory Distress Syndrome. II. A Longitudinal Study during the First 3 Weeks of Life
}

\author{
J. H. KOK, W. H. H. TEGELAERS, AND J. J. M. DE VIJLDER \\ Department of Neonatology and Department of Pediatrics, Academic Medical Center, University of Amsterdam, \\ Amsterdam, The Netherlands
}

\begin{abstract}
Serum thyroglobulin (Tg) and thyroid-stimulating hormone (TSH) levels were measured in 182 preterm and term infants. Samples were taken from cord blood, and at 1, 3, 7, 14, and 21 days after birth. The infants were divided into groups according to their perinatal characteristics: infants who were appropriate for gestational age, infants who were small for gestational age, and preterm infants who developed respiratory distress syndrome. These groups were subdivided according to gestation age. Tg serum levels showed a significant increase in the 1st day in all groups, and decreased significantly after about 1 wk. The highest $\mathrm{Tg}$ levels were found in the $1 \mathrm{st}$ wk of life in respiratory distress syndrome infants, and in infants with the lowest gestation ages. TSH levels increased at day 1 but only in appropriate and small for gestational age infants of more than 30 wk of gestation. TSH levels at day 1 in the groups with gestation ages of less than $30 \mathrm{wk}$ and in respiratory distress syndrome infants of more than 30 wk were low, reflecting a low TSH surge. We conclude that the neonatal increase of $\mathrm{Tg}$ is not merely caused by the TSH surge. We suggest that the $\mathrm{Tg}$ increase is due to an impaired degradation of $\mathrm{Tg}$, and/or to hemoconcentration, which are more pronounced in respiratory distress syndrome infants compared with appropriate for gestational age infants. (Pediatr Res 20: 10011003,1986 )
\end{abstract}

\section{Abbreviations}

Tg, thyroglobulin

TSH, thyroid-stimulating hormone

AGA, preterm infants with a birth weight appropriate for gestational age

SGA, term and preterm infants with a birth weight small for gestational age

RDS, preterm infants who developed respiratory distress syndrome

The adaptation of pituitary-thyroid function in the first weeks of life has been described extensively with respect to iodothyronines and TSH in term and preterm neonates (1-3). On the other hand, little information on $\mathrm{Tg}$ levels in the early postnatal period is available. Pezzino et al. (4) demonstrated an increase

Received April 10, 1985; accepted May 29, 1986

Correspondence J. J. M. de Vijlder, Academic Medical Center, Department of Pediatrics, Meibergdreef 9, 1105 AZ, Amsterdam, The Netherlands.

Supported by the Praeventie Fonds. of serum $\operatorname{Tg} 6 \mathrm{~h}$ after birth in term neonates. They supposed that this reflects the endogenous TSH stimulation immediately after birth. Black et al. (5) measured a postnatal increase of $\mathrm{Tg}$ in healthy term infants; in ill term and in preterm infants they could not demonstrate such an increase.

This study was undertaken to investigate the changes of $\mathrm{Tg}$ serum levels in infants with and without RDS during the first 3 wk of life in relation to TSH serum levels in the observed period.

\section{PATIENTS AND METHODS}

Patients. Venous blood samples were collected from 182 infants, when possible, at birth (cord 'lood), and at 1, 3, 7, 14, and 21 days after birth. Tg and TSIi serum concentrations of the samples were measured. In another paper (6) we demonstrated a dependence of $\mathrm{Tg}$ in cord serum on gestation age, birth weight, and RDS. Therefore these 182 infants were divided into groups according to their perinatal characteristics (AGA, SGA, and RDS, AGA infants who developed RDS). Each group was subdivided according to gestation age (I less than $30 \mathrm{wk}$; II 30-32 wk; III 33-36 wk; and IV more than 36 wk) (Table 1).

Definitions for gestation age and the RDS are given in our companion paper (6); the same exclusion criteria were used. In addition, infants who died during the observed period and infants who developed transient hypothyroidism after birth were excluded from this study. Infants with birth weights lower than the 10th percentile for their gestation age [according to Kloosterman's growth chart (7)] were considered SGA.

The study was approved by the Committee of Ethics of the Academic Hospital of the University of Amsterdam.

Methods. $\mathrm{Tg}$ and $\mathrm{TSH}$ levels were measured by radioimmunoassay methods (6). Due to the small volumes, $\mathrm{Tg}$ and $\mathrm{TSH}$ concentrations could not always be measured in all samples. In Figures 1 and 2 the number of samples in which determinations were performed is given for each sampling time.

All samples of one infant were assayed in the same run in order to avoid interassay variation.

Statistical analysis between groups of infants was performed with the use of Student's unpaired $t$ test, or analysis of variance. Comparisons within the groups were made by paired $t$ test; values were compared between cord and days 1,3 , and 21 , and between days 1 and 3 in all groups. Because of skewness of the distribution of the data the $\mathrm{Tg}$ and TSH values were analyzed after logarithmic transformation.

\section{RESULTS}

Table 1 shows the relevant clinical data concerning the infants in the different subgroups. Mean gestation ages in the youngest 
Table 1. Clinical data of the investigated groups for longitudinal studies

\begin{tabular}{|c|c|c|c|c|c|c|}
\hline & \multirow[b]{2}{*}{$n$} & \multicolumn{2}{|c|}{ Birth wt } & \multicolumn{2}{|c|}{ Gestational age } & \multirow{2}{*}{$\frac{\text { Sex }}{\text { Male/female }}$} \\
\hline & & Range & Mean $\pm S D$ & Range & Mean $\pm S D$ & \\
\hline AGA I & 15 & $890-1740$ & $1275 \pm 260$ & $26-29$ & $28.6 \pm 0.8$ & $7 / 8$ \\
\hline AGA II & 27 & $1160-2120$ & $1605 \pm 215$ & $30-32$ & $30.9 \pm 0.8$ & $18 / 9$ \\
\hline AGA III & 59 & $1570-3640$ & $2250 \pm 360$ & $33-36$ & $34.6 \pm 1.1$ & $32 / 27$ \\
\hline SGA II & 10 & $910-1285$ & $1055 \pm 135$ & $30-32$ & $31.7 \pm 0.5$ & $6 / 4$ \\
\hline SGA III & 21 & $890-2240$ & $1560 \pm 360$ & $33-36$ & $34.7 \pm 1.3$ & $16 / 5$ \\
\hline SGA IV & 19 & $1400-2350$ & $1990 \pm 280$ & $37-41$ & $37.7 \pm 1.2$ & $7 / 12$ \\
\hline RDS I & 18 & $810-1520$ & $1190 \pm 195$ & $26-29$ & $28.2 \pm 0.9$ & $9 / 9$ \\
\hline RDS II & 13 & $1190-1950$ & $1570 \pm 240$ & $30-32$ & $31.0 \pm 0.9$ & $8 / 5$ \\
\hline
\end{tabular}

AGA

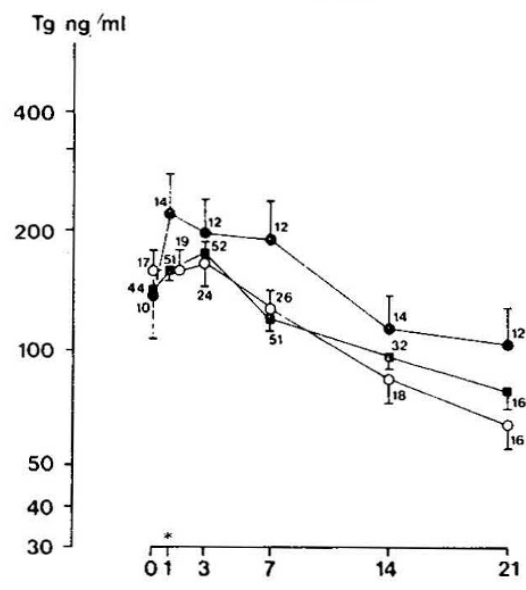

SGA

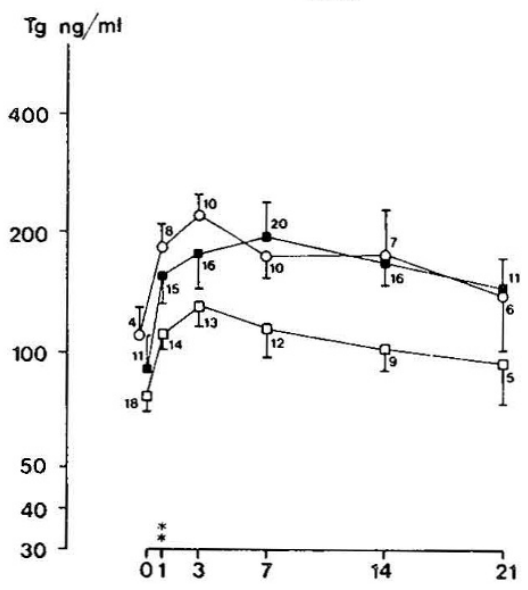

RDS

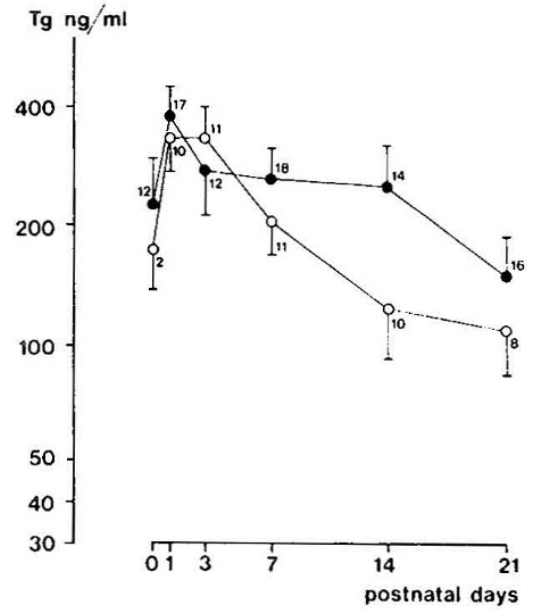

Fig. 1. Geometric means and SEM of Tg concentrations during the first 3 wk of life in AGA infants, SGA infants, and in infants who developed RDS. Samples at day 0 are taken from cord blood. Significance values between subgroups with different gestational ages are indicated by an asterisk

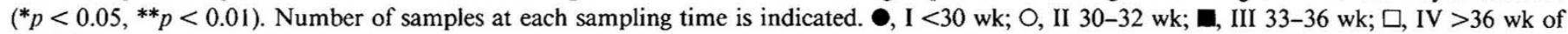
gestation.
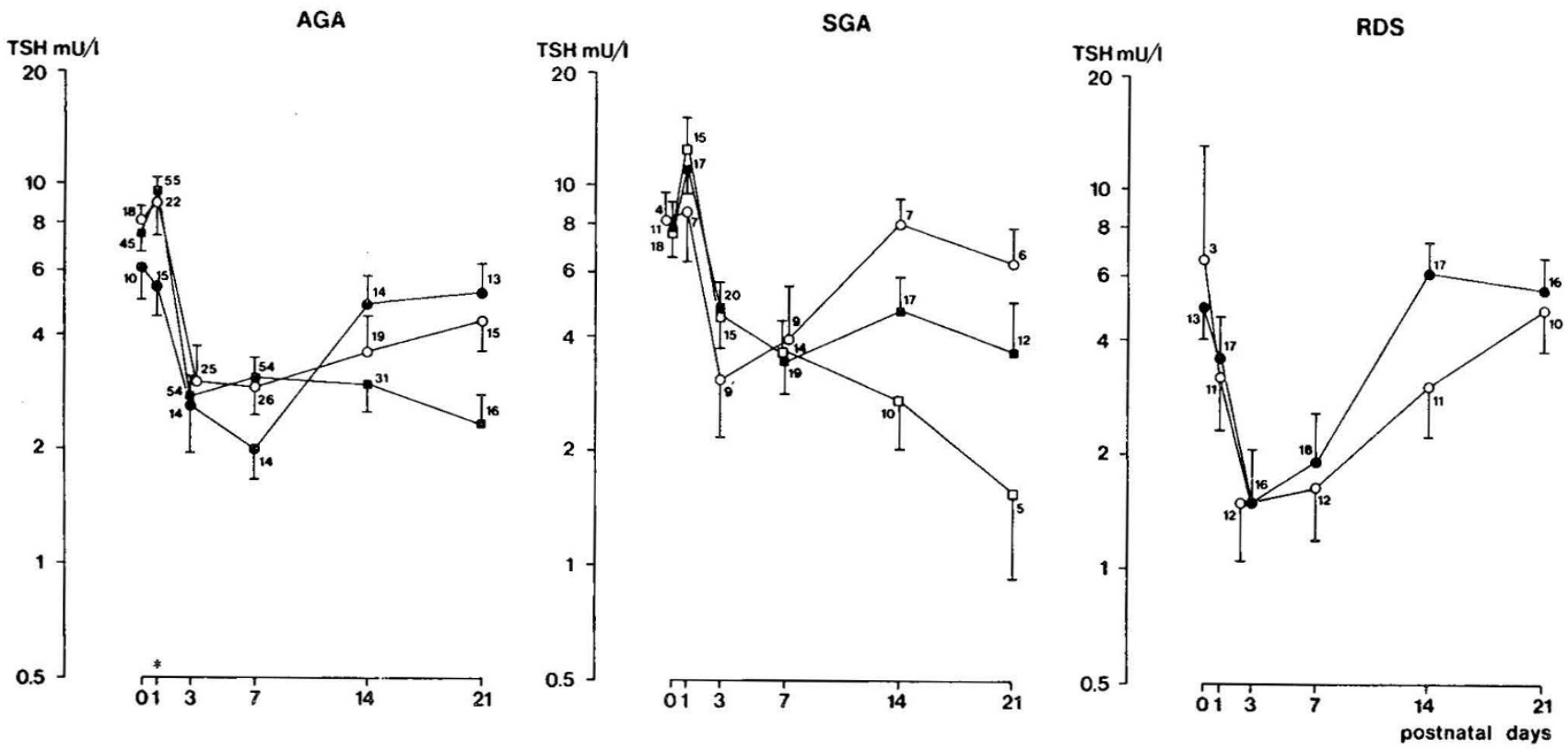

Fig. 2. Geometric means and SEM of TSH concentrations during the first $3 \mathrm{wk}$ of life in AGA infants, SGA infants, and in infants who developed RDS. Samples at day 0 are taken from cord blood. Significance values between subgroups with different gestational ages are indicated by an asterisk $\left({ }^{*} p<0.05,{ }^{* *} p<0.01\right)$. Number of samples at each sampling time is indicated. $\bullet$, I $<30 \mathrm{wk}$; O, II 30-32 wk; $\mathbf{\square}$, III 33-36 wk; $\square$, IV $>36$ wk of gestation. 
groups (AGA I and RDS I) are comparable, as is the case for AGA III and SGA III. However, in infants of 30-32 wk mean gestational age in SGA infants $(31.7 \pm 0.5 \mathrm{wk})$ is significantly higher than that in AGA or RDS infants $(30.9 \pm 0.8$ and $31.0 \pm$ $0.9 \mathrm{wk}$, respectively) $(p<0.05)$. SGA I and RDS III groups were not composed because of too small a number of infants (two and four, respectively).

Tg. All groups show a characteristic pattern of Tg serum levels in the first $3 \mathrm{wk}$ of life, as can be seen in Figure 1.

In all groups (except RDS II, because of short data in cord serum) a significant increase of $\mathrm{Tg}$ serum values is found 1 day after birth (AGA I, II, SGA II $p<0.05$; AGA III, SGA III, RDS I $p<0.01$; SGA IV $p<0.001$ ). Although in group AGA II mean $\mathrm{Tg}$ in cord serum and day 1 serum are the same, there is a significant increase of $\mathrm{Tg}$ in 13 infants of whom paired data were available. RDS infants show the highest mean $\mathrm{Tg}$ values in the first 7 days. In AGA II and III Tg serum levels at day 21 are significantly lower than cord serum levels $(p<0.001$ and $<0.01$, respectively). No differences between mean $\mathrm{Tg}$ levels on days 1 and 3 can be measured, except in SGA IV $(p<0.05)$.

Comparison of mean data between infants with the same perinatal characteristics but different gestation age shows that $\mathrm{Tg}$ values at day 1 are higher when gestation age is shorter (AGA I, II, III: $p<0.05$, SGA II, III, IV: $p<0.01$ : analysis of variance; RDS I, II: NS, $t$ test).

Differences between AGA, RDS, and SGA with comparable gestation ages are as follows. RDS I infants show higher $\mathrm{Tg}$ levels than AGA I during the period of observation. However, the difference is significant only on day $14(t$ test, $p<0.05)$. In the groups between $30-32$ wk, RDS II infants show significantly higher levels during the first 7 days (analysis of variance $p<$ 0.05 ), while SGA II infants show the highest levels after this period $(p<0.05)$. SGA infants show the highest levels after 1 wk in the groups between $33-36$ wk as well $(p<0.05)$.

$T S H$. The longitudinal pattern of TSH serum values in the investigated groups is shown in Figure 2. TSH levels of AGA I infants are significantly lower on day 1 than those of AGA II and III $(p<0.05)$. In SGA III and IV significantly higher TSH levels are found on day 1 compared with cord serum levels $(p<$ 0.01 and $<0.05$, respectively). In the groups between $30-32 \mathrm{wk}$ of gestation, RDS II infants show significantly lower TSH levels on day 1 than SGA II and AGA II infants (analysis of variance, $p<0.01)$. AGA I and RDS I infants show slightly but not significantly lower TSH levels on day 1 compared with cord serum. There are no significant differences in the course of TSH levels between AGA I and RDS I infants.

\section{DISCUSSION}

We found that $\mathrm{Tg}$ serum levels display a characteristic pattern in the first $3 \mathrm{wk}$ of life in all groups. Between 1-3 days after birth maximal $\mathrm{Tg}$ values were found. The highest $\mathrm{Tg}$ levels were observed in RDS infants. These findings are in contrast to those of Black et al. (5), who did not find differences between cord levels of $\mathrm{Tg}$ and $\mathrm{Tg}$ levels in the first $2 \mathrm{wk}$ of life in preterm infants. This is probably due to pooling of their data. De Nayer et al. (8) found high Tg cord serum concentrations and a decrease after the first weeks of life in preterm infants, in accordance with our data.

Pezzino et al. (4) suggested that the $\mathrm{Tg}$ increase after birth in term infants is caused by an endogenous TSH surge which can be demonstrated half an hour after birth (9). We did not measure the TSH surge, but others $(10,11)$ have shown a lower TSH surge in RDS infants than in healthy preterm infants of compa- rable gestation ages. Our finding that TSH concentrations on day 1 in RDS II infants are significantly lower than in AGA II or SGA II, probably reflects a low TSH surge in RDS infants. Because lower TSH levels were measured in both AGA I and RDS I infants on day 1, the TSH surge is probably low in the extreme premature infant as well. Therefore the TSH surge after birth cannot be the only factor responsible for the $\mathrm{Tg}$ increase after birth. This is particularly true in RDS infants, because these infants show the highest $\mathrm{Tg}$ values.

In addition to a given level of TSH stimulation, other factors leading to high $\mathrm{Tg}$ serum concentrations in preterm infants may include a higher permeability of the thyroid gland or a delayed degradation of $\mathrm{Tg}$ by an immature liver. A delayed degradation could be caused by a diminished binding of $\mathrm{Tg}$, resulting from an altered structure of the receptor, or from an altered composition of the carbohydrate chains of $\mathrm{Tg}$, as can also be found in thyroid cancer (12). Another factor may be the degree of hemoconcentration. Usually RDS infants show an increasing edema during the first days of life, with hypoalbuminemia (13) due to a higher capillary permeability. Since it is unlikely that $\mathrm{Tg}$ permeates the capillary wall (mol wt of Tg: 660,000) at the same rate as proteins of lower molecular weight, such as albumin, it is possible that the high Tg levels are due to hemoconcentration. However, in our study group mean hematocrit levels of RDS I and AGA I infants were not different during the 1 st wk.

In conclusion, $\mathrm{Tg}$ levels in preterm infants show a significant increase between 1 and 3 days after birth. This increase is not entirely attributable to the TSH surge. In preterm infants with RDS significantly higher $\mathrm{Tg}$ serum concentrations are found than in preterm infants who do not develop the syndrome.

Acknowledgment. The authors, thank Dr. J. G. Koppe, who provided the opportunity to collect the material; Mrs. H. Bikker, Dr. E. Endert, and the technicians of the laboratory of Endocrinology for Tg and TSH determinations; A. A. M. Hart for statistical supervision: and Dr. M. H. Gons for fruitful discussions.

\section{REFERENCES}

1. Fisher DA, Klein AH 1981 Thyroid development and disorders of thyroid function in the newborn. N Engl J Med 304:702-712

2. Jacobsen BB 1983 Thyroid function in infancy. Dan Med Bull 30:281-309

3. Uhrmann S, Marks KH, Maisels MJ, Friedman Z, Murray F, Kulin HE, Kapian M, Utiger R 1978 Thyroid function in the preterm infant: a longitudinal assessment. J Pediatr 92:968-973

4. Pezzino V, Filetti S, Belfiori A, Proto S, Donzelli G, Vigneri R 1981 Serum thyroglobulin levels in the newborn. J Clin Endocrinol Metab 52:364-366

5. Black EG, Bodden SJ, Hulse JA, Hoffenberg R 1982 Serum thyroglobulin in normal and hypothyroid neonates. Clin Endocrinol 16:267-274

6. Kok JH, De Vijlder JJM, Tegelaers WHH 1986 Serum thyroglobulin levels in infants with and without respiratory distress syndrome. I. Cord blood study. Pediatr Res 20:000-000

7. Kloosterman GJ 1970 On intrauterine growth. Int J Gynaecol Obstet 6:895

8. De Nayer Ph, Cornette C, Vanderschueren M, Eggermont E, De Vlieger H, Jaecken J, Beckers C 1984 Serum thyroglobulin levels in preterm neonates. Clin Endocrinol 21:149-153

9. Fisher DA, Odell WD 1969 Acute release of thyrotropin in the newborn. J Clin Invest 48:1670-1677

10. Cuestas RA, Engel RR 1979 Thyroid function in preterm infants with respiratory distress syndrome. J Pediatr 94:643-646

11. Klein AH, Foley B, Kenny FM, Fisher DA 1979 Thyroid hormone and thyrotropin responses to parturition in premature infants with and without the respiratory distress syndrome. Pediatrics 63:380-385

12. Ikekubo K, Pervos R, Schneider AB 1980 Clearance of normal and tumorrelated thyroglobulin from the circulation of rats: role of the terminal sialic acid residues. Metabolism 29:673-681

13. Hardie G, Harrison VC, Kench JE 1968 Further observations on serum proteins in respiratory distress syndrome of the newborn. Arch Dis Child 43:471-474 